EPJ Web of Conferences 66, 03032 (2014)

DOI: $10.1051 /$ epjconf/ 20146603032

(C) Owned by the authors, published by EDP Sciences, 2014

\title{
Probing the symmetry energy at low density using observable from neck fragmentation
}

E. De Filippo ${ }^{1, a}$, L. Acosta ${ }^{2}$, C. Agodi ${ }^{2}$, F. Amorini ${ }^{2}$, L. Auditore ${ }^{3}$, V. Baran ${ }^{4}$, I. Berceanu ${ }^{5}$, T. Cap ${ }^{6}$, G. Cardella ${ }^{1}$, M. Colonna ${ }^{2}$, L. Francalanza ${ }^{7,2}$, E. Geraci ${ }^{7,1}$, R. Gianì ${ }^{7,2}$, L. Grassi ${ }^{2}$, A. Grzeszczuk $^{8}$, P. Guazzoni ${ }^{9}$, J. Han ${ }^{2}$, E. La Guidara ${ }^{1}$, G. Lanzalone ${ }^{10,2}$, I. Lombardo ${ }^{11}$, C. Maiolino ${ }^{2}$, T. Minniti ${ }^{3}$, A. Pagano ${ }^{1}$, E.V. Pagano ${ }^{7,2}$, M. Papa ${ }^{1}$, E. Piasecki ${ }^{6,12}$, R. Płaneta ${ }^{13}$, S. Pirrone ${ }^{1}$, G. Politi ${ }^{7,1}$, A. Pop ${ }^{5}$, F. Porto ${ }^{7,2}$, L. Quattrocchi ${ }^{3}$, F. Rizzo ${ }^{7,2}$, E. Rosato ${ }^{11}$, P. Russotto ${ }^{1}$, K. Siwek-Wilczyńska ${ }^{6}$, I Skwira-Chalot ${ }^{6}$, A. Trifirò ${ }^{3}$, M. Trimarchi ${ }^{3}$, G. Verde ${ }^{1}$, M. Vigilante ${ }^{11}$, J. Wilczyński ${ }^{12}$, and L. Zetta ${ }^{9}$

${ }^{1}$ INFN Sez. di Catania, Italy

2 INFN, Laboratori Nazionali del Sud, Catania, Italy

${ }^{3}$ INFN, Gruppo coll. di Messina and Dip. di Fisica, Università di Messina, Italy

${ }^{4}$ Physics Faculty, University of Bucharest, Romania

${ }^{5}$ National Institute of Physics and Nuclear Engineering "Horia Hulubei", Bucharest, Romania

${ }^{6}$ Faculty of Physics and Heavy lon Laboratory, University of Warsaw, Poland

${ }^{7}$ Dipartimento di Fisica e Astronomia, Univ. di Catania, Italy

${ }^{8}$ Institute of Physics, University of Silesia, Katowice, Poland

${ }^{9}$ INFN, Sezione di Milano and Dipartimento di Fisica, Univ. di Milano, Italy

10 "Kore" Università, Enna, Italy

${ }^{11}$ INFN, Sezione di Napoli and Dipartimento di Fisica, Univ. di Napoli, Italy

${ }^{12}$ National Centre for Nuclear Research, Otwock-Świerk, Poland

${ }^{13}$ M. Smoluchowski Inst. of Physics, Jagellonian Univ., Cracow, Poland

\begin{abstract}
Semi-peripheral collisions of ${ }^{64} \mathrm{Ni}+{ }^{124} \mathrm{Sn}$ in direct and inverse kinematics, at $35 \mathrm{~A} \mathrm{MeV}$ incident energy, are studied to characterize the dynamical origin and alignments properties of fragments emission at midrapidity. This emission is partly due to the fragmentation of a transient neck joining the projectile and target in the early stages of the reaction. Recently we exploited observable from neck fragmentation to constraint the density dependence of the symmetry energy. In this contribution we focus on the density evolution of the mid-rapidity source. An experimental survey is suggested to check if data are consistent with the formation of neck fragments in a dilute density region in contact with the projectile-like and target-like spectators at normal saturation density.
\end{abstract}

\section{Introduction}

In the Fermi energy regime (10-100 A MeV) semi-peripheral collisions are characterized by binary reactions where projectile and target nuclei experience a substantial overlap of matter. In these reactions, beside the production of excited projectile-like (PLF) and target-like (TLF) nuclei, a complex

\footnotetext{
ae-mail: defilippo@ct.infn.it
} 
dynamical rearrangements of nucleons in the participant region within a short time scale $(<50 \mathrm{fm} / \mathrm{c})$ can take place. Transport model calculations indicate the formation of a neck-like structure at subnormal baryonic density [1]. Intermediate mass fragments (IMF) are mainly the remnants of this structure formed during the stage of reseparation of the PLF and TLF. The neutron enrichment of the neck originating IMFs, observed experimentally, is interpreted by transport model calculations as a migration of neutrons against protons due to the density gradient [2] between the dilute neck region and the PLF and TLF residues. The nucleons transport process trough the neck depends upon the isovector part of the mean field interaction, thus carrying important information on reaction dynamics and symmetry energy that is a key ingredient for dynamical models of heavy ion collisions and astrophysical predictions [3, 4]. In a recent paper [5], studying the reactions ${ }^{112,124} \mathrm{Sn}+{ }^{58,64} \mathrm{Ni}$ at $35 \mathrm{~A}$ $\mathrm{MeV}$ with the $4 \pi$ detector CHIMERA, we have correlated the isotopic composition of IMFs emitted at midrapidity (up to charge $\mathrm{Z} \leq 8$ ) in semiperipheral collisions with their emission timescale: IMFs emitted in the early stage of the reaction show strong angular anisotropies and large values of $N / Z$ isospin asymmetry when they are compared with those produced in sequential statistical emission. Together with results of Ref. [6], where light charged particles $(Z=1,2)$ and light IMFs $(Z=3,4)$ are taken into account, and Ref. [7] a coherent scenario of neutron rich particle emission at midrapidity correlated with early emission time and kinematical properties of the fragments can be traced. In Ref. [5] we compared the data for dynamically emitted neck fragments with a Stochastic Mean Field simulation (SMF) $[1,2]$, getting indication for a linear behavior of the EOS symmetry potential at subsaturation density. In this contribution we firstly show how to disentangle between statistical and dynamical emitted particles at midrapidity. Finally we check, starting from experimental data and in comparison with SMF model, of what extend data can support the scenario of formation of neck fragments in a dilute density region in contact with the PLF and TLF at normal saturation density.

\section{Experimental details and results}

The direct kinematic experiment has been performed at the Catania INFN-LNS superconducting cyclotron. Beams of ${ }^{64} \mathrm{Ni}$ and ${ }^{58} \mathrm{Ni}$ at $35 \mathrm{~A} . \mathrm{MeV}$ impinged on self-supporting ${ }^{124} \mathrm{Sn}$ and ${ }^{112} \mathrm{Sn}$ thin targets whose thickness are respectively $187 \mu \mathrm{g} / \mathrm{cm}^{2}$ and $268 \mu \mathrm{g} / \mathrm{cm}^{2}$. Reaction products were detected with the $4 \pi$ detector CHIMERA. This experiment exploited in a large number of the CHIMERA Si-Csi detectors, the silicon pulse shape technique [8], giving the possibility to identify in charge the particles that are stopped in the silicon detectors. We have selected almost complete events where the total charge was $45 \leq Z_{T O T} \leq 80$ and the parallel momentum of the colliding system was at least $60 \%$ of the total one. Semi-pheripheral collisions were selected gating on the total charged particle multiplicity $M \leq 7$. We selected ternary events in the final state, where a PLF and TLF residue and one IMF ( $3 \leq Z \leq 20)$ were simultaneously present.

A way to study the dynamical origin and alignment of mid-rapidity fragments is to consider the angle $\theta_{P R O X}$ between the TLF and IMF-PLF center of mass direction $\left(\mathbf{n}_{s}=\mathbf{V}_{P L F-I M F}^{c . m}-\mathbf{V}_{T L F}\right)$ and the break-up axis defined as the relative velocity between the PLF and IMF oriented from the light to heavy fragment. In the left panel of Fig. 1 for the ${ }^{64} \mathrm{Ni}+{ }^{124} \mathrm{Sn}$ system the blue hashed histogram distribution for $\mathrm{Z}=6$ shows a strong enhancement when $\cos \left(\theta_{P R O X}\right)>0.6$, indicating a backward IMF emission with respect to the PLF with a strong alignment along the PLF-TLF separation axis. We have extended this procedure considering also the TLF break-up. The separation between PLF emission or TLF emission is chosen gating by the relative velocities due to the mutual PLF-IMF or TLF-IMF Coulomb repulsion respectively. Conventionally the sign of $\cos \left(\theta_{P R O X}\right)$ for the TLF decay is taken such a way that the peak at $\cos \left(\theta_{P R O X}\right)<-0.6$ represents the aligned break-up of $\mathrm{Z}=6 \mathrm{IMF}$ towards midrapidity for TLF break-up. In Fig. 1 the distribution for IMFs of charge $Z=6$ for the target-like 

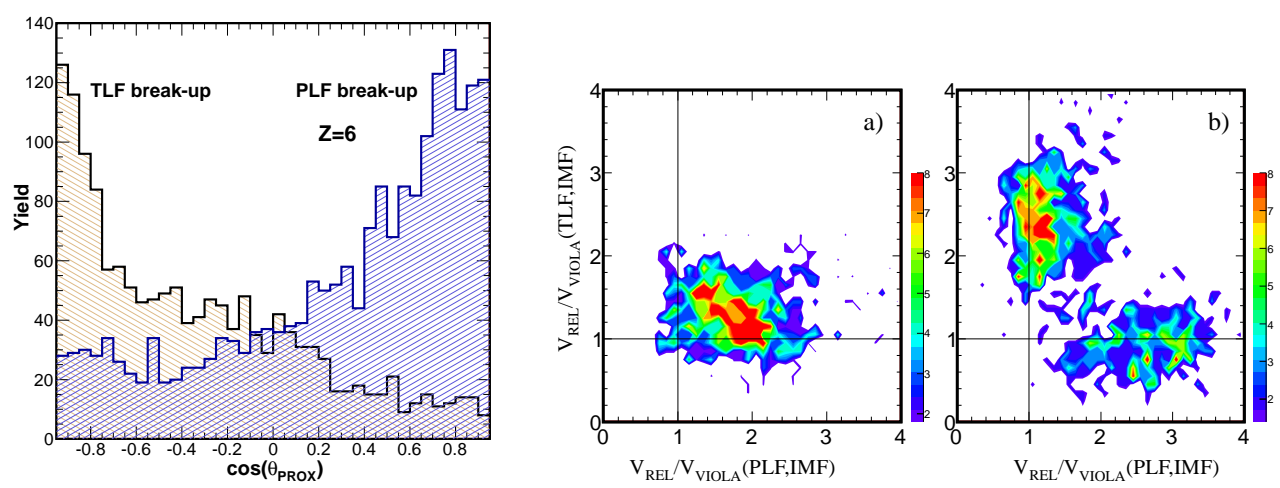

Figure 1. Left hand panel: Angular distribution $\cos \left(\theta_{\text {PROX }}\right)$ for $\mathrm{Z}=6$ IMFs respect to the PLF (blue hashed histogram) and respect to the TLF (brown hashed histogram), for ${ }^{64} \mathrm{Ni}+{ }^{124} \mathrm{Sn}$ reaction (see text). Right hand panel: correlations between relative velocities $V_{R E L} / V_{\text {Viola }}$ for $\mathrm{Z}=6$ gating with a) $\cos \left(\theta_{\text {PROX }}\right)<0.6$ for PLF $\left(\left(<-0.6\right.\right.$ for TLF) break-up; b) gating with $\cos \left(\theta_{P R O X}\right)<0$ for PLF ( $>0$ for TLF) break-up respectively.

(Sn-like) is shown as the brown hashed histogram. The sum of the two contributes to the dynamical IMF emission at midrapidity. The fragment-fragment relative velocities $r_{1}=V_{R E L}(P L F, I M F)$ and $r_{2}=V_{R E L}(T L F, I M F)$ were calculated and are reported in Figs. 1a,b) (right panel) normalized to the velocity $\left(V_{\text {Viola }}\right)$ corresponding to their mutual Coulomb repulsion. Fig. 1a) shows the correlation $r_{1}-r_{2}$ obtained gating on $\cos \left(\theta_{P R O X}\right)>0.6$ for PLF break-up and $\cos \left(\theta_{P R O X}\right)<-0.6$ for TLF break-up. This condition selects values of $r_{1}$ and $r_{2}$ larger than unity indicating IMFs of dynamical origin in prompt ternary division. Fig. 1b) shows the opposite condition. Here the values $r_{1}=1$ and $r_{2}=1$ indicate rispectively the loci for a sequential decay of IMFs from respectively the PLF and TLF (see Refs. [5, 9] for details). We have used these selection methods to careful disentangle the pattern of dynamically emitted fragments respect to statistically emitted fragments for both reverse and direct kinematical reactions studied here. Fig. 2 (left panel) shows for a SMF simulation of $6 \mathrm{fm}$ impact parameter in the reaction ${ }^{124} \mathrm{Sn}+{ }^{64} \mathrm{Ni}$ at $35 \mathrm{~A} \mathrm{MeV}$ the time evolution of the density in a box around midrapidity region in ternary events. The plot shows a plateau at around $\rho / \rho_{0}=1 / 3$ giving indication for the formation of a low density neck region in the midrapidity region in a short time scale. From the experimental point of view, as a first step investigation, we have considered data from the same ${ }^{124} \mathrm{Sn}$ $+{ }^{64} \mathrm{Ni}$ at $35 \mathrm{~A} \mathrm{MeV}$ and we have used the method described above in order to distinguish between dynamically emitted fragments from statistically emitted fragments. Fig. 2b,d) (right panel) give an example of results for this selection for $\mathrm{Z}=4$ IMFs respectively for dynamically emitted fragments $b$ ) and sequential emitted fragments from PLF d).

Considering the approximation of a three body system we have: $E_{T O T}^{c . m}=E_{1}+E_{2}+E_{3}=$ $p_{1-23}^{2} / \mu_{1-23}+p_{23}^{2} / \mu_{23}=E_{1-23}+E_{23}$ where the index 1,2,3 refer respectively to the IMF,PLF and TLF. $E_{1-23}$ is the relative energy of the IMF (1) respect to the PLF-TLF (23) center of mass system and $E_{23}$ is the energy of the relative motion of the PLF-TLF system. The distribution of the ratio between $E_{1-23}$ and the kinetic energy relative to the Coulomb repulsion $E_{\text {coul }}$ is shown in Fig. 2c) for $\mathrm{Z}=4$ statistically emitted particles, and, as expected, is well centered around one and is used as validation of the method. In Fig. 2a) we have done the calculation for $\mathrm{Z}=4$ dynamically emitted particles estimating the Coulomb energy $E_{\text {coul }}\left(d / r_{0}\right)$, where $r_{0}=1.2 \mathrm{fm}$ and $\left(d / r_{0}\right) A^{1 / 3}$ is the IMF radius, in two different configurations: the first one corresponding to a compact configuration $d=r_{0}$ 

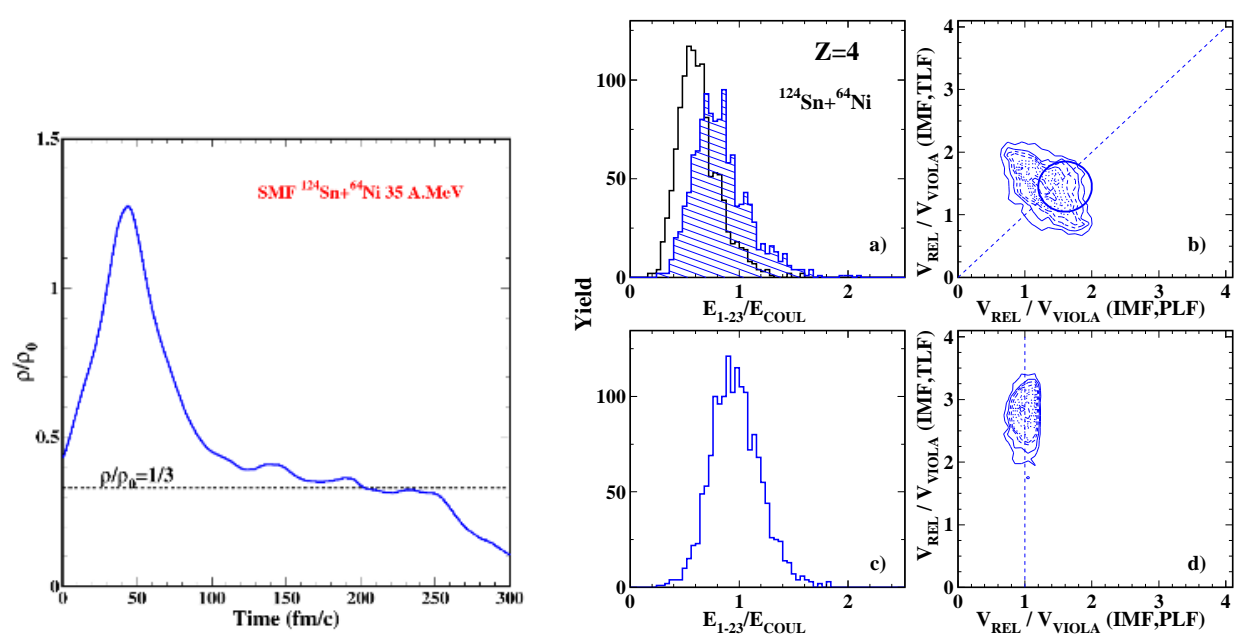

Figure 2. (left panel): SMF simulation at $6 \mathrm{fm}$ showing the time evolution of the density in ternary events. (right panel) a) ratio of $E_{1-23} / E_{\text {coul }}$ for $\mathrm{Z}=4$ dynamically emitted fragments for $E_{\text {coul }}$ calculated in a compact (empty histogram) and in dilute (filled histogram) configuration. c) $E_{1-23} / E_{\text {coul }}$ for sequential $\mathrm{Z}=4$ emitted fragments. b,d) $V_{R E L} / V_{\text {Viola }}$ plots for $\mathrm{Z}=4$ dynamically and sequentially emitted fragments respectively.

(empty histogram) and the second one assuming a dilute configuration (filled histogram) corresponding to about $\rho_{=} 0.051 / \mathrm{fm}^{3}$ resulting from the average $\rho$ values of the SMF calculation. Results show that experimental data are consistent with the formation of neck fragments in a dilute density region. The analysis of the direct kinematics reaction show the same behaviour. Of course, more detailed investigations are needed in order to validate this simplified approach.

In conclusion, we have studied with the $4 \pi$ detector CHIMERA the two reactions ${ }^{64,58} \mathrm{Ni}+{ }^{124,112} \mathrm{Sn}$ and ${ }^{124,112} \mathrm{Sn}+{ }^{64,58} \mathrm{Ni}$ at the same energy of relative motion $(35 \mathrm{~A} \mathrm{MeV})$ and defined new methods to use the neck fragmentation mechanism as an observable to constraints the symmetry energy term of nuclear EOS at subsaturation densities [5]. In this contribution we have presented a phenomenological estimation of the density in ternary neck events in order to probe that, as stated by transport model calculations, neck fragments are formed in presence of a density gradient.

\section{References}

[1] V. Baran, M. Colonna and M. Di Toro, Nucl. Phys. A 730, 329 (2004).

[2] J. Rizzo et al., Nucl. Phys. A 806, 79 (2008).

[3] M.B. Tsang et al., Phys. Rev. C86, 015803 (2012).

[4] J.M. Lattimer, Annu. Rev. Nucl. Part. Sci. 62, 485 (2012).

[5] E. De Filippo et al., Phys. Rev. C 86, 014610 (2012).

[6] Z. Kohley et al., Phys. Rev. C 86, 044605 (2012).

[7] S. Hudan et al., Phys. Rev. C 86, 021603 (2012).

[8] M. Alderighi et al. IEEE Trans. Nucl. Science, 52, 1624 (2005).

[9] E. De Filippo et al., Journal of Physics CS 420, 012105 (2013). 